\title{
L'ingénierie hydraulique d'EDF à l'international : une nouvelle donne
}

\author{
J.-M. Devernay *, X. Cailmail ** \\ * Engineering and Construction Division, ** Generation and Transmission Division \\ EDF Ingénierie hydraulique
}

\section{INTRODUCTION}

* A sa création, il y a près de 50 ans, Electricité de France voit son histoire se confondre avec celle de l'aménagement hydroélectrique de la France aujourd hui réalisé à plus de $90 \%$. Au travers de la réalisation et de l'exploitation de l'un des plus grands ensembles hydroélectriques du monde, Electricité de France a acquis des compétences exceptionnelles dans le domaine de l'ingénierie, de l'environnement, de l'exploitation et de la gestion des ressources en eau.

Mais l'histoire de l'hydraulique dans le monde ne saurait être achevée alors qu'un homme sur deux sur notre planète ne dispose pas d'électricité et que dans de nombreux pays le potentiel hydró́lectrique reste exploité à moins de $10 \%$.

C'est ce nouveau défi, la construction du parc hydroélectrique mondial, que l'entreprise Electricité de France, avec les bailleurs de fonds et ses partenaires électriciens et industriels, doit relever en s'appuyant sur son expérience et ses moyens financiers."

C"est, en substance, par ces mots de Jean-Michel Fauve, Directeur des Affaires Internationales d'Electricité de France que s'ouvre le dernier numéro du magazine de l'ingénierie hydraulique d'EDF «Architectes de l'Eau".

Ce faisant, Jean-Michel Fauve souligne bien la place et l'importance de la contribution des Architectes de l'Eau d'Electricité de France au service du développement international de leur entreprise, en parfaite continuité avec leurs missions de maintien et de renforcement du patrimoine hydroélectrique français.

Les quelques exemples présentés dans l'article qui suit illustrent la diversité des réponses apportées par l'ingénierie hydraulique d'Electricité de France pour contribuer à l'essor international de l'entreprise, tant dans des actions d'ingénierie que dans des partenariats industriels au sein desquels EDF intervient en tant qu'investisseur et opérateur.

Ce sont ces projets qui constituent désormais la nouvelle donne du métier sans frontière des Architectes de l'Eau d'Electricité de France auprès de leurs clients et de leurs partenaires.

\section{L'INGÉNIERIE HYDRAULIQUE D'EDF À L'INTERNATIONAL}

Potentiel hydraulique, croissance de la consommation d'électricité, évolution du système électrique, besoins de financement, constituent aujourd'hui autant de critères susceptibles de sous-tendre les interventions de l'ingénierie hydraulique d'EDF à l'international.

Poursuivant et élargissant l'action conduite depuis plusieurs décennies, Electricité de France met son expérience au service de ses clients et partenaires sur la scène internationale dans les domaines d'expertise et d'intervention privilégiée qui sont les siens et qui concernent tout particulièrement :

- l'ingénierie de conception et de réalisation d'ouvrages neufs,

- l'ingénierie d'exploitation, de maintenance et de réhabilitation,

- et depuis quelques années les prises de participation et la gestion de sociétés.

Ces interventions peuvent se faire selon des modalités très variées pouvant aller de la prestation de services en tant qu'ingénieur-conseil jusqu'à la Maîtrise d’Euvre complète et au partenariat industriel en tant qu'investisseur et opérateur dans des sociétés de patrimoine.

Cette diversité des projets et de leur taille, des prestations et des rôles dévolus à EDF constituent autant d'opportunités pour les Architectes de l'Eau d'EDF d'exercer leur métier multiple au service de leurs clients et auprès de leurs partenaires.

C'est ce métier multiple qui, de la conception à la réalisation, des évaluations technico-économiques à la mise en service et à l'exploitation, des ouvrages neufs à la réhabilitation des ouvrages anciens, de la conduite à la maintenance des ouvrages, est présenté au travers des projets décrits ci-après.

Ces quelques projets, qui ne peuvent à eux seuls englober toutes ses spécialités, illustrent cependant les aspects les plus significatifs et les plus récents de l'internationalisation de l'ingénierie hydraulique d'EDF avec:

- Nam Theun 2 : investissement, conception et exploitation d'une centrale hydraulique de $600 \mathrm{MW}$ au Laos.

- Rio Diamante et Los Nihuiles : investissement et production d'électricité en Argentine.

- Les études des projets de Station de Transfert d'Energie par Pompage de Khiritharn en Thaïlande et de Matenggeng en Indonésie.

- L'ingénierie des ouvrages hydrauliques conventionnels de Vinh' Son au Vietnam et de Garafiri en Guinée.

- L'expertise et la sécurité des ouvrages existants en Inde (Orissa) avec le projet "Dam Safety » ainsi que dans de nombreux autres pays du monde. 
- L'optimisation et la rénovation des ouvrages existants en Inde avec le projet de Kalinadi et de Victoria Falls en Zambie.

- La maîtrise de l'eau pour l'irrigation avec le projet des Doukkala au Maroc.

Ces projets illustrent également le rôle que les Architectes de l'Eau d'EDF entendent jouer au service du développement international de leur entreprise en la confortant dans son métier d'investisseur et d'opérateur d'ouvrages de production hydraulique.

\section{III $\square$ EDF INVESTISSEUR, ARCHITECTE INDUSTRIEL ET OPÉRATEUR AU LAOS AVEC LE PROJET DE NAM THEUN 2}

Pour la première fois en tant que producteur, EDF a l'ambition de s'implanter durablement au Laos grâce au projet de Nam Theun 2 (600 MW) dont la mise en service est prévue en 1999.

A l'origine de ce projet, un protocole signé entre les gouvernements laotiens et thaillandais prévoit la fourniture, par le Laos, de $1500 \mathrm{MW}$ à Energy Generating Authority of Thailland (EGAT). La Thaillande a en effet des besoins croissants de puissance électrique alors que le fort potentiel hydroélectrique du Laos est encore faiblement utilisé.

Le développement du projet est financé par apport de fonds propres par un groupe de 5 partenaires investisseurs qui, outre EDF, comprend trois sociétés thaîlandaises, le constructeur Italian-Thaï Development (ITD), le financier Phatra, le spécialiste de la télécommunication Jasmine et l'ensemblier australien Transfield.

L'accord entre les 5 partenaires attribue à EDF les études de définition du projet et l'exploitation pendant 25 ans de la future centrale, dont les ouvrages et l'exploitation seront transférés au gouvernement laotien, Maître d'Ouvrage final, à l'issue de cette période. L'australien Transfield est chargé, avec Italian-Thaï et Jasmine, de la réalisation des travaux qui doivent démarrer fin 1995.

Responsable des études de définition du projet, EDF Ingénierie Hydraulique, en concertation avec d'autres spécialistes d'EDF (géologues, topographes, développeurs,

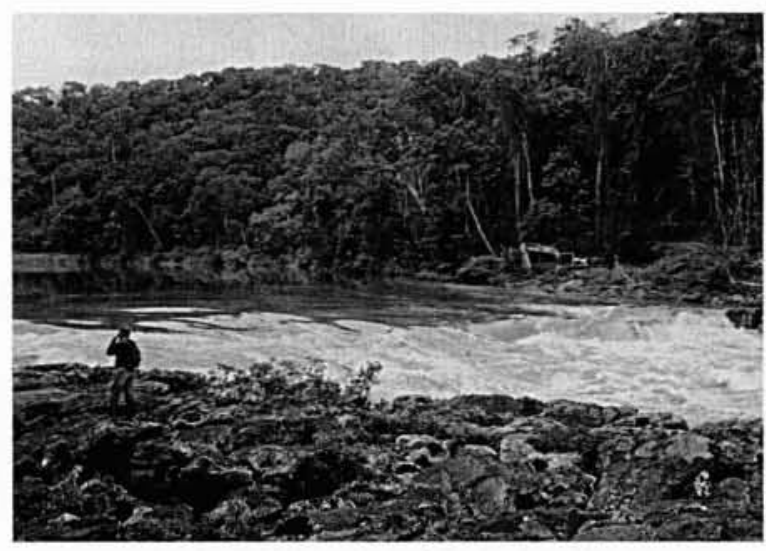

1. Nam Theun : site du barrage. financiers, juristes et exploitants) s'emploie à mener à bien dans les délais impartis en 1995 les travaux de reconnaissances, l'étude de projet détaillé et la rédaction des spécifications, ainsi que l'étude d'impact sur l'environnement.

Pour EDF, entreprise intégrée, le projet de Nam Theun 2, qui signifie engagement dans la production hydroélectrique au Laos et fourniture d'électricité à la Thaỉlande, est une mise en application majeure de la stratégie de développement international de l'Entreprise. Dans le cadre de ce projet, les Architectes de l'Eau démontrent leurs capacités à réaliser les études de projets internationaux et à conforter EDF dans son rôle d'investisseur et d'opérateur.

\section{IV $\square$ EDF INVESTISSEUR ET PRODUCTEUR D'ÉLECTRICITÉ EN ARGENTINE AVEC LES OUVRAGES DE RIO DIA- MANTE ET LOS NIHUILES}

En remportant en 1994 les appels d'offres pour l'achat de plus de la moitié des actions des complexes hydroélectriques de Rio Diamante (400 MW) et Los Nihuiles (300 MW), EDF est devenu producteur d'électricité en Argentine, dans la province de Mendoza au nord ouest de Buenos Aires, une région en forte croissance et plaque tournante entre l'Argentine et le Chili.

Ces installations, distantes de $30 \mathrm{~km}$ et dont la gestion est désormais assurée sous la direction de trois spécialistes d'EDF, doivent permettre à EDF de produire 1,5 milliard de kWh par an.

C'est en association avec une banque d'affaires argentine, Banco de Galicia, et un investisseur local qu'EDF a obtenu la majorité des actions de ces deux complexes hydroélectriques. La part d'EDF au sein de ce consortium représente environ 40 millions de dollars pour un investissement total de 120 millions de dollars.

Après la prise de participation en 1992 dans la société de distribution d'Electricité du nord de la capitale argentine (Edenor) et avec celle prise également en 1994 dans la société de transport (Distrocuyo), ce nouvel investissement est significatif de la volonté de développement d'EDF à l'international.

Il est également significatif du rôle de l'hydraulique dans ce développement pour les besoins duquel se conjuguent investissement financier conséquent, investissement humain pour approfondir la connaissance des systèmes électriques, et investissement stratégique pour préparer l'avenir.

\section{$V \square$ CONCEPTION DES STATIONS DE TRANSFERT D'ÉNERGIE PAR POM- PAGE EN THAÏLANDE ET EN INDONÉ- SIE}

Forts de leur expérience française de conception et d'exploitation de 13 Stations de Transfert d'Energie par Pompage (STEP) totalisant près de $5000 \mathrm{MW}$, les Architectes de l'Eau d'EDF sont devenus au fil des ans l'un des leaders de cette technologie dans le cercle restreint des ingénieries mondiales. 


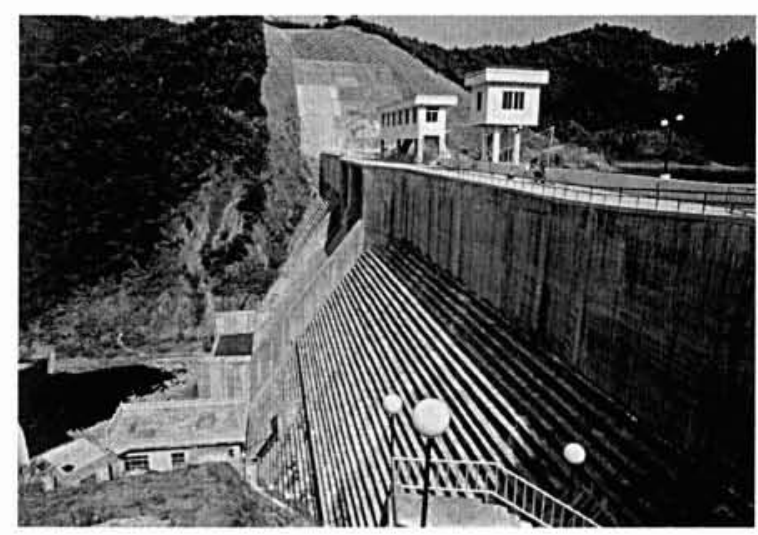

2. Conghua-Guangzhou : barrage inférieur.

Ainsi EDF Ingénierie Hydraulique intervient, ou est intervenue ces dernières années, sur des projets en Chine (Conghua-Guangzhou I (1 $200 \mathrm{MW})$, Tianhuangping (1 $800 \mathrm{MW})$ ), en Inde (Bhira $150 \mathrm{MW}$ ), en Pologne (Mloty $750 \mathrm{MW}$ ), et en Algérie (Ighil Emda $150 \mathrm{MW}$ ).

A ces projets, sont venus s'ajouter en 1993 et 1994, les projets de Khiritharn $(650 \mathrm{MW})$ en Thaillande, de Matenggeng ( $1000 \mathrm{MW})$ en Indonésie, de Yangyang ( $1000 \mathrm{MW}$ ) en Corée et de Pu Shi He (1 $200 \mathrm{MW})$ en Chine qui confortent la présence de l'ingénierie hydraulique d'EDF en Asie.

\subsection{La STEP de Khiritharn en Thailande}

Dans un pays où les besoins en Electricité augmentent de $15 \%$ par an et où les centrales hydroélectriques n'assurent que $10 \%$ de la production, le contexte était particulièrement favorable pour qu'Electricity Generating Authority of Thailland (EGAT) décide d'engager un important programme d'études et de réalisation de stations de transfert d'énergie par pompage.

Identifié par EGAT en 1991, le site de Khiritharn est rapidement apparu propice, à partir d'une retenue existante, à la réalisation d'une STEP mettant à profit une chute de $350 \mathrm{~m}$. C'est la faisabilité de ce projet dont la mise en service est prévue en 2004 qu'EDF, en partenariat avec SOGREAH, réalise pour le compte d'EGAT.

Compte tenu notamment des contraintes d'intégration au réseau électrique et d'impact sur l'environnement, c'est une variante équipée à $650 \mathrm{MW}$ et permettant une production annuelle de $1650 \mathrm{GWh}$ qui a été finalement recommandée à l'issue de l'évaluation économique d'un total de 13 configurations du projet variant de 600 à $2000 \mathrm{MW}$.

Bien entendu les relations de confiance instaurées avec EGAT et la connaissance pointue du système électrique Thaï acquise au cours de ces études par les ingénieurs hydrauliciens d'EDF ont permis de conforter les décisions d'EDF investisseur dans le cadre du projet de Nam Theun 2 au Laos dont la justification économique et la rentabilité sont directement liées à la structure et aux évolutions à venir du réseau thaílandais dans son ensemble.

\subsection{La STEP de Matenggeng sur l'île de Java}

L'importante augmentation de la consommation en Indonésie et à Java (6\% par an) va nécessiter la mise en service de nouveaux moyens de production $(10000 \mathrm{MW}$ installés en 93 pour $35000 \mathrm{MW}$ prévus en 2005). Ces moyens seront essentiellement thermiques compte tenu du faible potentiel naturel équipable par des ouvrages hydrauliques gravitaires « classiques ».

Le recours au pompage-turbinage pour assurer la souplesse d'exploitation du réseau est donc devenu un point de passage obligé pour l'entreprise nationale de production, transport et distribution d'électricité indonésienne, le Perusahaan Umum Listrik Negara (PLN).

C'est dans ce cadre qu'une dizaine d'ingénieurs d'EDF Ingénierie Hydraulique viennent d'engager sur place, en collaboration avec PLN, les études de faisabilité de la STEP de Matenggeng d'une puissance installée de $1000 \mathrm{MW}$ qui utilisera une dénivelée de $500 \mathrm{~m}$ entre 2 réservoirs à créer.

Avec cette première étude de projet hydraulique, les Architectes de l'Eau d'EDF contribuent à la consolidation du partenariat avec PLN, premier client à l'étranger d'EDF, hors nucléaire.

\section{VI $\square$ CONCEPTION ET PARTICIPATION À LA RÉALISATION D'OUVRAGES DE PRODUCTION HYDROÉLECTRIQUE AU VIETNAM ET EN GUINÉE}

Confrontés à la croissance de leurs besoins en énergie électrique, de nombreux pays font le choix de mettre en valeur leur potentiel hydroélectrique lorsqu'ils peuvent mobiliser, sur leurs propres ressources ou avec l'aide des bailleurs de fonds, les moyens financiers nécessaires.

Que le projet soit réalisé entièrement sur fonds propres, comme celui de Vinh Son au Vietnam, ou développé avec le soutien des bailleurs de fonds multilatéraux, comme celui de Garafiri en Guinée, les Architectes de l'Eau d'EDF s'engagent auprès de leurs clients pour accompagner à long terme leur stratégie de développement de la production d'énergie.

\subsection{Le projet de Vinh Son au Vietnam}

Pour pallier le déséquilibre entre une production hydraulique excédentaire dans le nord du pays et une production thermique déficitaire dans le centre et le sud du pays, le Ministère vietnamien de l'Énergie a décidé à la fin des années 80 la réalisation du projet de Vinh Son (66 MW).

Sur ce projet, EDF Ingénierie Hydraulique assure la conception et la maîtrise d'œuvre pour le compte du Maître d'Ouvrage, la Compagnie de Production $\mathrm{n}^{\circ} 3$ (PC3). Parmi la dizaine d'ingénieurs d'EDF qui ont participé à ce projet, deux ont été détachés sur le site pour conseiller le Client et superviser les travaux.

En complément de la conception et de la réalisation de l'ouvrage, EDF assure la formation du personnel de la future centrale à la technologie du contrôle-commande et s'implique de fait dans l'exploitation à venir de cette centrale hydraulique d'un type jusque-là unique au Vietnam. 


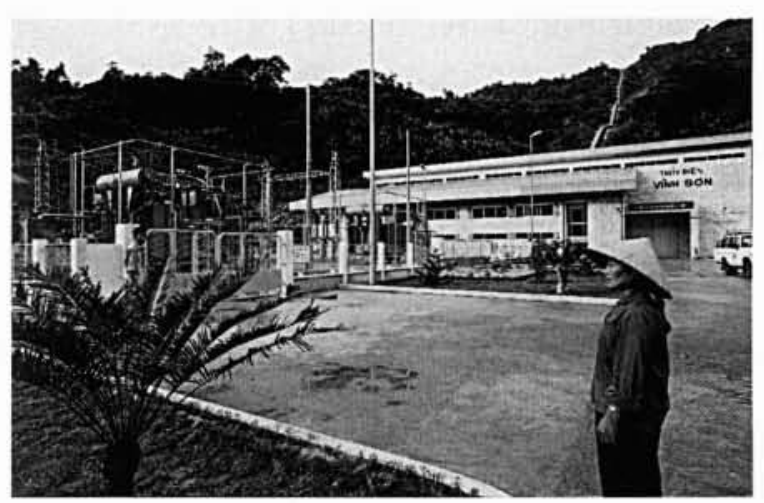

3. Usine de Vinh'Son.

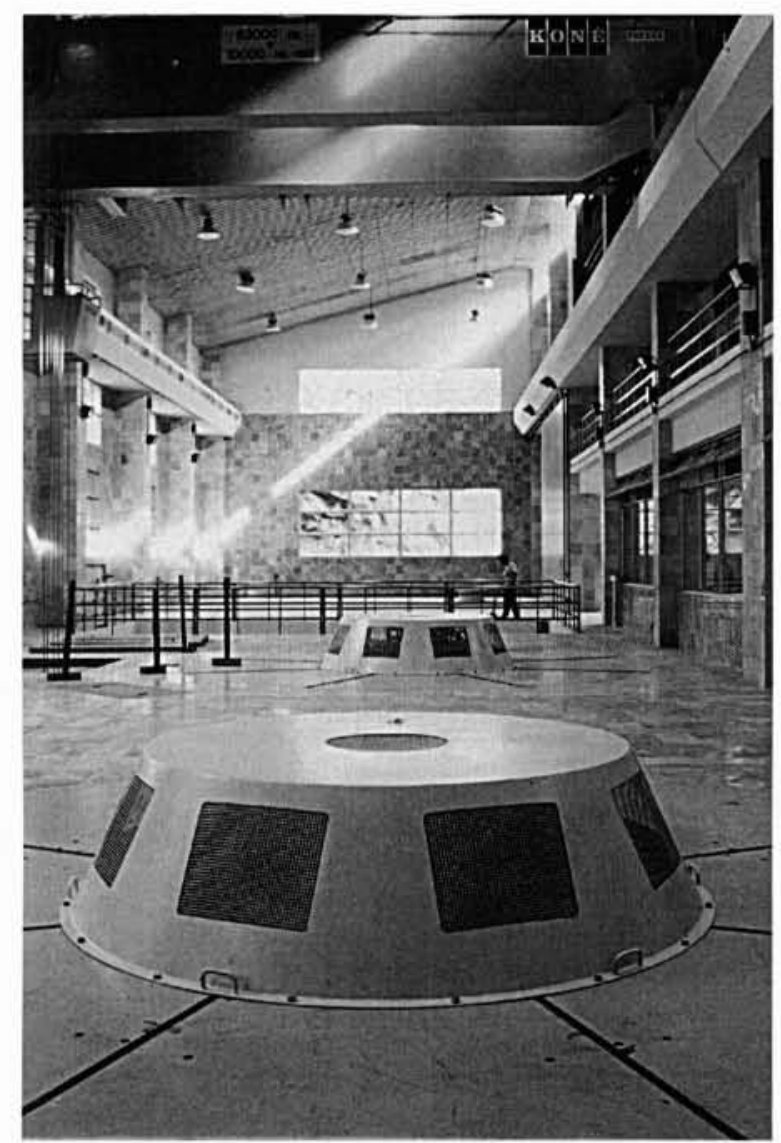

4. Vinh'Son : Salle des machines.

\subsection{Le projet de Garafiri en Guinée}

L'enjeu du projet de Garafiri est de taille pour le Gouvernement Guinéen puisque les délestages dus aux arrêts pour entretien des groupes thermiques et au manque d'eau à l'étiage pour les centrales hydrauliques entraînent un important déficit de production d'environ $100 \mathrm{GWh} / \mathrm{an}$, soit le tiers de la consommation potentielle.

A l'issue des études d'inventaire démarrées en 1987, puis des études comparatives réalisées en 1988, c'est le complexe hydroélectrique Garafiri-Kaléta qui a été retenu pour être le moyen de production à réaliser en priorité. Le projet de Garafiri est la pièce principale de ce complexe avec une puissance installée de $75 \mathrm{MW}$ pour un produc- tible moyen de $264 \mathrm{GWh} / \mathrm{an}$. Etudes d'avant-projet détaillé puis préparation des documents d'appels d'offres vont se poursuivre en 1995 avec l'attribution des marchés de travaux et l'engagement de la réalisation.

Electricité de France, associée au bureau d'études Coyne et Bellier, a participé pour le compte du Ministère des Ressources Naturelles des Energies et de l'Environnement de Guinée (MRNEE) à la totalité des études du projet de Garafiri qui, contrairement à bien d'autres projets africains, est un projet neuf identifié pour la première fois en 1988.

C'est de ce même Groupement Coyne et Bellier - Electricité de France que le MRNEE a sollicité une offre pour assurer la maîtrise d'œuvre partielle de la construction de l'aménagement de Garafiri qui doit être financée par les principaux bailleurs de fonds (Caisse Française de Développement, Banque Européenne d'Investissement auxquels se joindront d'autres organismes financiers arabes et canadiens) aux côtés de l'Etat Guinéen.

\section{EXPERTISE ET SÉCURITÉ DES OUVRAGES EXISTANTS EN INDE ET DE PAR LE MONDE}

Avec l'expérience de la conception, de la réalisation, de l'exploitation et de la maintenance de 220 grands barrages en France, la compétence de l'ingénierie hydraulique d'EDF pour l'expertise, la surveillance, la réévaluation de la sécurité et la réhabilitation des ouvrages existants s'exprime bien au-delà des frontières de l'hexagone.

En Inde, EDF renforce sa présence avec la signature d'un contrat pour la réévaluation de la sécurité de huit barrages classés «à risques" dans l'Etat d'Orissa. En association avec le bureau d'études India Kirloskar Consultants, EDF Ingénierie Hydraulique apporte son expertise pour des études portant notamment sur la stabilité, l'évacuation des crues et l'auscultation et propose des mesures de réhabilitation adaptées.

Ce contrat s'inscrit dans le vaste programme « Sécurité des Barrages - Dam Safety " financé par un prêt de la Banque Mondiale.

L'auscultation s'exporte bien et les récentes prestations d'EDF au Gabon, en Côte d'Ivoire, en Chine, au Mexique et ailleurs en attestent. Qu'il s'agisse, pour ne citer que ceux-là, de Madagascar pour une revue générale de l'organisation en matière d'auscultation, du Maroc pour une

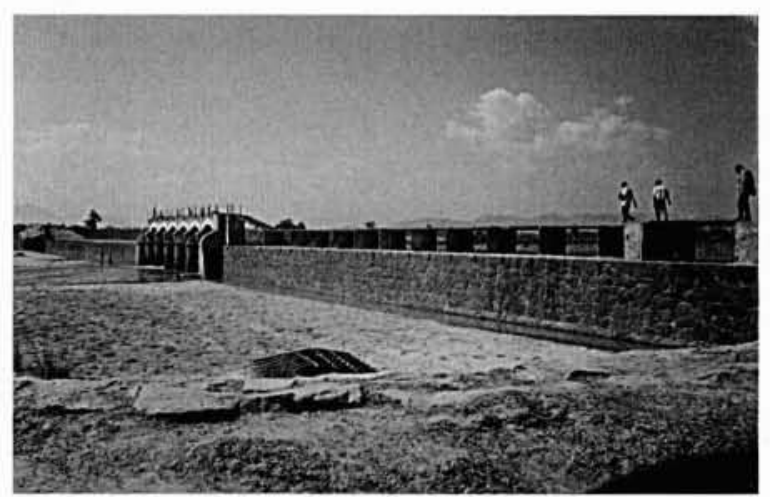

5. Barrage de SORADA. 
analyse du comportement d'un ouvrage ou de la Roumanie pour la mise en place d'un plan de formation, tous ces contrats soulignent et confortent la notoriété de l'ingénierie hydraulique d'EDF dans le domaine de la maîtrise de la sécurité des barrages.

\section{D OPTIMISATION ET MODERNISA- TION DES OUVRAGES EXISTANTS EN INDE ET EN ZAMBIE}

La maîtrise de l'eau, c'est assurer la gestion de la ressource en eau dans toutes ses dimensions : gérer le passage des crues pour un ensemble d'aménagements d'un même bassin versant, optimiser les différents usages de l'eau dont la production énergétique d'un ensemble d'usines hydroélectriques, rénover des anciennes centrales pour accroître la production.

Pour EDF, exploitant de ses propres ouvrages, ces objectifs majeurs sont prioritaires.

C'est à la satisfaction de ces mêmes objectifs que les Architectes de l'Eau d'EDF contribuent auprès de leurs clients étrangers, qu'il s'agisse de l'installation de postes de conduite automatique centralisée, de l'utilisation de modèles de prévision des débits, de propagation de débits et de régulation de niveaux ou de la rénovation d'un système de contrôle-commande.

Ainsi en Inde, la compétence d'EDF pour la gestion optimisée a été sollicitée dans le Karnataka, l'un des états les plus industriels de l'Inde. Avec ce projet, EDF a mis son savoir-faire au profit de la gestion d'un ensemble d'aménagements situés sur la rivière Kali comprenant 6 centrales d'une puissance totale de $1640 \mathrm{MW}$ et sept réservoirs de capacité totale d'environ 5 milliards de $\mathrm{m}^{3}$. Logiciels de prévision de débits et poste centralisé de conduite permettront une gestion en temps réel de la vallée en permettant les ajustements nécessités par les besoins du réseau, compte tenu des contraintes d'exploitation.

En Zambie, c'est à la modernisation des usines des chutes Victoria (108 MW) sur le Zambèze, l'un des plus grands fleuves de la planète, que l'ingénierie hydraulique d'EDF contribue à l'issue d'un appel d'offres international. Expertise du contrôle commande, des prises d'eau, des vannes de tête, du poste haute tension et du poste centralisé de commande, propositions d'améliorations (notamment pour résoudre d'importants problèmes de vibrations) et spécifications techniques associées doivent se traduire, après mise en cuvre, par une augmentation d'au moins $10 \%$ du productible.

\section{IX — MAÎTRISE DE L'EAU POUR L'IRRIGA- TION AU MAROC}

C'est dans la région des Abda Doukkala, que l'Office Régional de Mise en Valeur Agricole des Doukkala (ORMVAD) a entrepris la réalisation d'un vaste périmètre d'irrigation qui devra doubler à terme la surface de 61000 ha déjà irriguée.

Après avoir déjà réalisé les études des ouvrages d'amenée et de relevage destinés à alimenter $120 \mathrm{~km}$ de canaux, EDF ingénierie hydraulique s'est vu confier par I'ORMVAD une mission d'assistance pour la réalisation des ouvrages d'amenée (galerie de $13 \mathrm{~km}$ de longueur et de $6,40 \mathrm{~m}$ de diamètre).

Dans le cadre de ce contrat, les experts d'EDF (topographes, géologues-géotechniciens, génie-civilistes) interviennent aux côtés de deux ingénieurs d'EDF mis à la disposition de l'ORMVAD pour toute la durée des travaux.

Au Maroc, les Architectes de l'Eau d'EDF partagent leur savoir-faire de la Maîtrise de l'Eau. Puisque maîtriser l'eau pour l'irrigation, c'est également contribuer de façon décisive au développement. 